\title{
Machine Learning from Garden Path Sentences: The Application of Computational Linguistics
}

\author{
http://dx.doi.org/10.3991/ijet.v9i6.4109 \\ J.L. Du ${ }^{1}$, P.F. $\mathrm{Yu}^{1}$ and M.L. $\mathrm{Li}^{2}$ \\ ${ }^{1}$ Guangdong University of Foreign Studies, Guangzhou, China \\ ${ }^{2}$ Griffith University, Australia
}

\begin{abstract}
This paper discusses the application of computational linguistics in the machine learning (ML) system for the processing of garden path sentences. ML is closely related to artificial intelligence and linguistic cognition. The rapid and efficient processing of the complex structures is an effective method to test the system. By means of parsing the garden path sentence, we draw a conclusion that the integration of theoretical and statistical methods is helpful for the development of ML system.
\end{abstract}

Index Terms-machine learning, computational linguistics, processing breakdown, backtracking, garden path sentences

\section{INTRODUCTION}

Machine learning (ML) focuses on the creation of algorithms used to help computers to evolve behaviors on the basis of empirical data. It is related to the computational applications, including data mining programs to find the general rules in large data sets and information filtering systems to automatically learn users' interests.

ML is closely related to software and artificial intelligence (AI). It highlights the rapid and effective applications of decision making in the domains of engineering and computational linguistics. A lot of topics have been discussed recently. Sometimes, the ill-conditioning of hidden layer output matrix and the complexity of singular value decomposition can prevent the further development of ELM [1]. The useful approach can result in the compact network classifiers with the characters of fast response and robust prediction accuracy on unseen data [2].

The behavioral analysis is a helpful idea for ML development. Controlling complex dynamic systems requires skills by which operators can demonstrate rather than completely describe.

The transferring human control skill to an automatic controller, e.g. the behavioral cloning, is becoming another focus of ML [3]. Both statistical-based and logic-based techniques are effective. The statistical method is accurate despite of its poor interpretation. The logic approach is easy to understand but hard to obtain accurate result for engineering applications. The special CAQ can combine the continuous and discrete values, and deal with the continuous attributes without forcing them into a discrete representation, which leads to more efficient concept formation [4].

The advancement of classification skill improves ML effectiveness. The classification of spectral data and other high-dimensional data plays an important role in ML domain. The principal component analysis (PCA) can reduce high-dimensional spectral data and improve the predictive performance of ML skills by means of the classification of high-dimensional data [5]. The emergent technology is good at performance in regression and large dataset classification applications. The special system, ELM for classification, is less sensitive to user specified parameters, and it can achieve better generalization performance than traditional system [6]. ML algorithm highlights the effectiveness of integration. For example, CLIP4, a hybrid inductive ML algorithm, is effective in generation of rules that involve inequalities and in production of rules from subsets stored at the leaf nodes. CLIP4 has built-in features, e.g. tree pruning and methods for partitioning the data, generates model of data consisting of well-generalized rules, and ranks attributes used for feature selection [7]. The weighted shortest processing time rule, the earliest due date rule and Moore's algorithm can construct an optimal schedule for the problem to minimize functions respectively [8].

A lot of neural networks are helpful for ML development. For example, the dropout prediction method for elearning courses is proved to be effective. It is based on a kind of ML technique which is feed-forward neural networks. It is used in vector machines, and the overall accuracy, sensitivity and precision, and its results were found to be significantly better than those reported ever [9]. It shows the fact that rich interaction between users and ML system is feasible for both user and machine. Thus the user can better interact with the ML system, share intelligence, and further trust the system. Simultaneously system is improved with the deep processing of behavioral analysis. The potential of rich human-computer collaboration via on-the-spot interactions is becoming a promising direction for ML systems [10].

The effective ML models involved in the language processing will be discussed below.

\section{ILG AND Berns’ Machine LEARning System}

An effective ML is a system which tries to partly eliminate the need for human intuition in data processing. However, ML cannot entirely eliminate the influence of human intuition. It is important for a designer to decide which data should be represented and what mechanisms should be included in the system by means of the processing of the data. The functional analysis of phrase structure and lexical transfer may be a useful approach for ML [11]. The effective system can bridge the language gap by mediating the communication despite the fact that the technology may not be always perfect, and bring the high user satisfaction and interaction efficiency [12]. For 
the purpose of reducing the translation ambiguities and generating grammatically correct and fluent translation output, the linguistic knowledge is necessary and normal [13]. ML is a research on computational approaches to learning, including some results on computational methods applied to learning problems.

ML focuses on the automatical learning to recognize complex patterns and on the making of intelligent decisions. Sometimes it is difficult for ML system to work since the set of all possible behaviors may be so complex that system fails to describe clearly in programming languages. Ilg and Berns creates the ML system which comprises the characters of evaluation, internal evaluation, adaption, stochast-action-offset, prototypical actions, action elements, actions, actors, self-organizing representation of the state space, sensors, and external reward. Please see the figure 1.

In the ML system, Ilg and Berns [14] think that the core is the realization of the action elements and the internal evaluation function. Sensors and actors are put outside the domain of system even though both are closely related to the action elements and the internal evaluation.

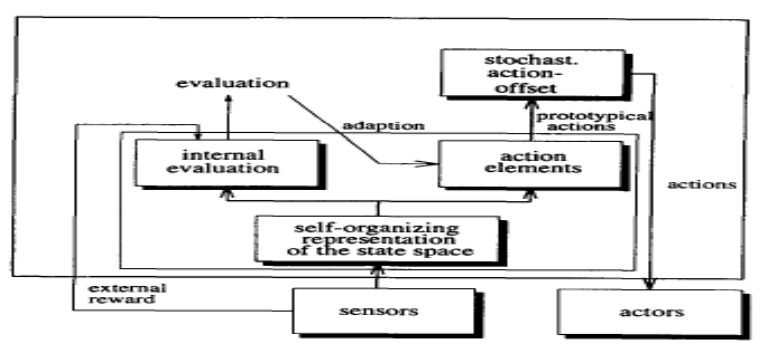

Figure 1 Ilg and Berns' ML System

Sensors firstly give the influence to self-organizing representation of the state space, and simultaneously bring the external reward to internal evaluation.

A critic element can generate a reward for the actual state. An action element can determine the next control values. An adaptation of both components is made in each control step. The self-organization of the state space is necessary and unavoidable. Self-organizing representation of the state space continues to affect the processing result of internal evaluation and action elements. Internal evaluation is a process whose next steps are evaluation and adaption. Both internal evaluation and action elements, under the supervision of prototypical actions, step towards stochast-action-offset, whose response is transmitted to actors by means of actions. The learning architecture is proved effective for adaptive control especially on the basis of reinforcement learning.

Based on the Ilg and Berns' model, we can find that the skill of computational linguistics is helpful for processing in ML. The processing of garden path sentences which have the complex structures will be discussed below.

\section{Machine LeARning For LANGUAge Processing OF GARDEN PATH SENTENCES}

The discussed below comprises the structural processing, the statistical analysis and the algorithm processing of garden path sentence.

\section{A. The Structural Analysis of Garden Path Sentences}

A garden path sentence is a complex one which includes the original pseudo processing and the ultimate genuine processing. Backtracking and processing breakdown are the peculiarities. Generally speaking, the sentence is grammatically parsed as a correct one after the misinterpretation is backtracked. That means the reader is lured by the pseudo analysis into a parse that turns out to be a dead end. "Garden path" means "to be misled", and the decoders sometimes are "led down the garden path".

A garden path sentence may be either a simple sentence whose syntactic structure is simple and whose main structure comprises a few clauses (Example 1), or a complex sentence which has the complex syntactic structure (Example 2).

Example 1 Time flies like an arrow; fruit flies like a banana.

Example 2 The horse raced past the barn fell.

The Example 1 is a simple garden path sentence, in which "flies" shifts from a verb to a subject noun, and "like" changes from a preposition to a verb.

The Example 2 is a complex garden path sentence. In the original processing, readers consider "raced past the barn" to be the structure of active intransitive verb plus "past the barn". This is the pseudo processing. However, with the advancement of processing, readers find that "raced past the barn" is only a reduced relative clause with a passive participle. This is the genuine and successful processing.

The processing of garden path sentences is hard for both human beings and machines. The integration of various computational technologies, e.g. CFG, RTN, WFST, and CYK algorithm, can help machine learning systems fully understand the natural language processing. The multiple processing is as follows.

Example 3 The complex houses married and single students and their families.

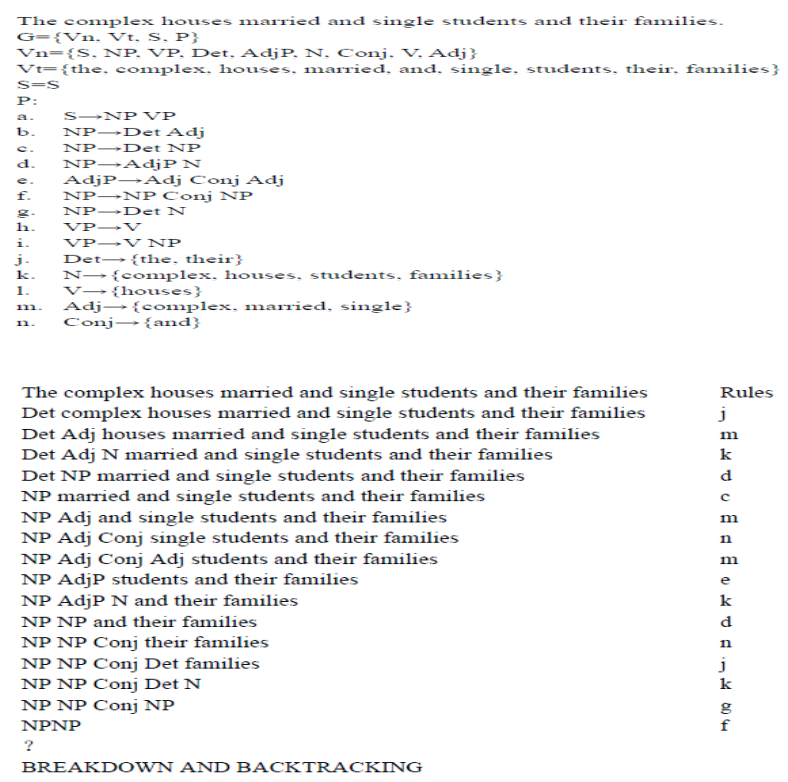

The pseudo decoding above shows that NP+NP structure is not the ultimate processing, which leads to the processing breakdown and system has to backtrack to the point where "houses" is considered to be a verb rather 
than a noun plural. The ultimate decoding is provided below.

According to the analysis, we can obtain a clear tree diagram in which "the complex" is regarded as NP; "houses", V; "married and single students and their families", NP.

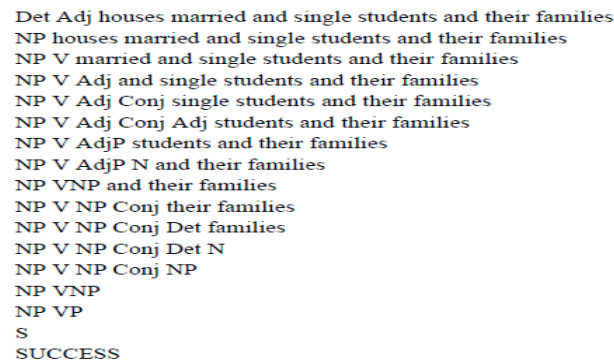

with the involvement of breakdown and backtracking is a non-well-formed sub-string table in which not all elements are successfully parsed. On the contrary, the structure of genuine processing is a well-formed sub-string table in which the ultimate symbol " $S$ " is obtained and all the elements are decoded. The non-well-formed sub-string table is as follows.

In Table 1, we can find that there is no ultimate symbol " $S$ ", which means the processing is not well-formed. The framework of processing is NP+NP. Please see the table from the pseudo processing.

The well-formed substring table is an $n^{*} n$ matrix in which $n$ refers to the string of length.

$\operatorname{Chart}(i, j)=\left\{A \mid A \rightarrow{ }^{*} W_{i+1} \ldots W_{j}\right\}$

Input sentence:

${ }_{0}$ The. ${ }_{1}$ complex. ${ }_{2}$ houses. ${ }_{3}$ married. 4 and . ${ }_{5}$ single. 6 students . 7 and. stheir. 9 families. 10

TABle 1 The Matrix OF PSEUdo PROCESSING

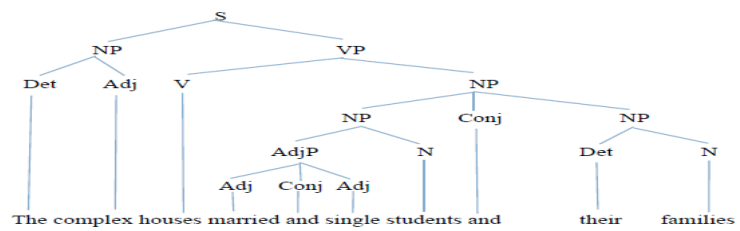

Figure 2 The Tree Diagram of Example 3

A recursive transition network can be created by the processing of Example 3 to present the whole procedure of pseudo and genuine processing of the garden path sentence.

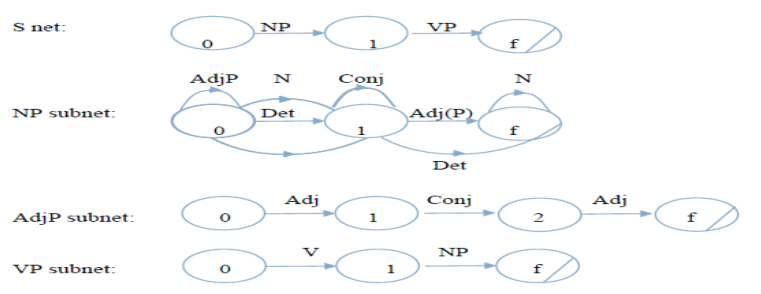

Figure 3 The Recursive Transition Network of Example 3

The recursive transition network above comprises $\mathrm{S}$ net, NP subnet, AdjP subnet, and VP subnet. The created framework can be used to decode the Example 3 effectively, including the pseudo and genuine processing.

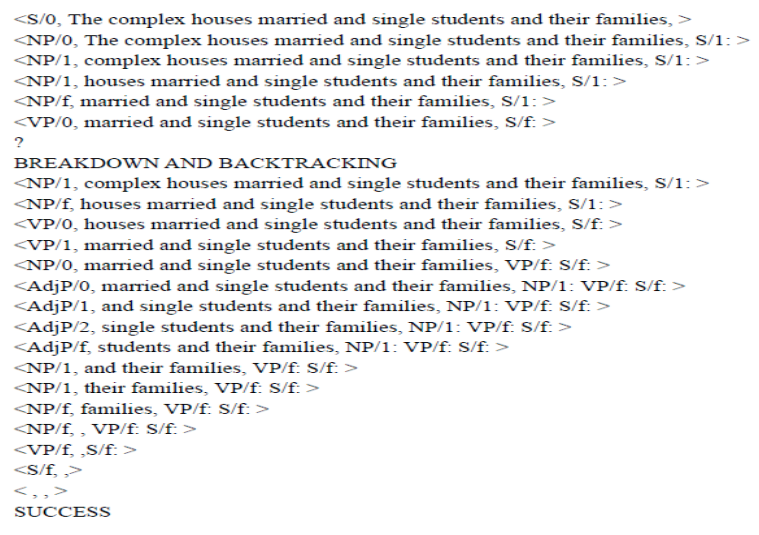

The processing above shows that the preferred structure of "houses" as a noun plural is not a genuine choice. The option in which "houses" is considered a verb is chosen as the acceptable choice after the processing breakdown and backtracking. The framework from the pseudo processing

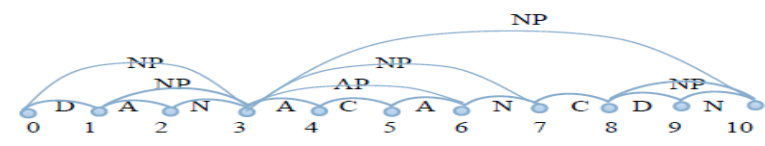

Figure 4 Sub-String Table of Pseudo Processing for Example 3

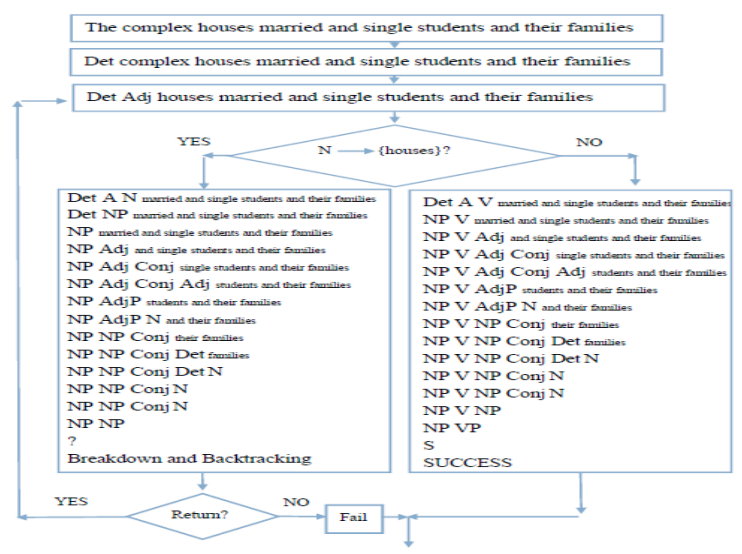

Figure 5 The Processing Flowchart of Example 3

In Figure 4, the whole framework is not a successful structure since there is no rule for $\mathrm{S} \rightarrow \mathrm{NP}$ NP. That means the processing is not a perfect one, and breakdown and backtracking may be involved.

In Figure 5, the crucial processing is the choice of "houses". When the rule $\mathrm{N} \rightarrow$ \{houses\} is chosen, the left processing is stimulated (the statistical evidence will be provided in 3.2). The result of processing is NP+NP, indicating the appearance of breakdown and backtracking. The system returns to the point where the verb category of "houses", namely $\mathrm{V} \rightarrow$ \{houses\}, is selected.

Based on the theoretical analysis, we will analyze Example 3 by means of statistical data to show the reason why the effect of processing breakdown and backtracking come into being [15]. 


\section{Machine Learning From Garden PATH Sentences: The ApPlication of Computational Linguistics}

\section{B. The Statistics-Based Analysis of GP Sentences}

In Example 3, the key word is "house" which can be considered to be either a verb or a noun. The choice with much higher frequency is the preferred structure from the cognitive and statistical perspective. In statistics, according to the significant difference of frequency, the structure can be divided into preferred and unpreferred structures. In the corpus (http://bncweb.lancs.ac.uk/) which includes 98,313,429 words, the "houses (verb)" has 215 hits and the "houses (noun)" has 4784 hits if the model "houses + node +any verb (any noun)" is chosen. Please see the statistical analysis of the significance difference.

$$
\text { Formula 1: } x^{2}=\sum \frac{(O-E)^{2}}{E}
$$

TABLE 2 THE NONPARAMETRIC STATISTICS OF "HOUSES"

\begin{tabular}{|l|l|l|l|l|l|}
\hline Category & 0 & $\mathrm{E}$ & $0-\mathrm{E}$ & $(0-\mathrm{E})^{2}$ & $(0-\mathrm{E})^{2} / \mathrm{E}$ \\
\hline Houses. verb & 215 & 2499.50 & -2284.50 & $\begin{array}{l}5218940 . \\
25\end{array}$ & 2087.99 \\
\hline Houses. noun & 4784 & 2499.50 & 2284.50 & $\begin{array}{l}5218940 . \\
25\end{array}$ & 2087.99 \\
\hline \multicolumn{5}{|c|}{$X^{2}$} & \\
\hline
\end{tabular}

In Table 2, Chi-square test value is $x^{2}$; $O$ means observed frequency; $E$ means expected frequency. If the significance level is .05; degree of freedom is 1 ; then critical value is 3.84 .

$$
\mathrm{X}^{2}=4175.99, \mathrm{P}<.05
$$

The statistical result shows that the structure in which "houses" is considered to be a noun is a preferred structure.

The structure of "the complex houses" can be statistically analyzed (http://bncweb.lancs.ac.uk/) by means of the formulas shown below: $\mathrm{r}(\mathrm{x})=\mathrm{r}(\mathrm{the})=6041234$; $\mathrm{r}(\mathrm{y})=$ $\mathrm{r}($ complex $)=9381 ; \mathrm{r}(\mathrm{z})=\mathrm{r}($ houses $)=9822 ; \mathrm{r}(\mathrm{x}, \mathrm{y})=$ $\mathrm{r}($ the, complex $)=1080 ; \mathrm{r}(\mathrm{y}, \mathrm{z})=\mathrm{r}($ complex, houses $)=4$.

$$
\begin{aligned}
& \text { Formula 2: } \\
& \qquad p(y \mid x)=\frac{p(x, y)}{p(x)}=\frac{r(x, y)}{r(x)}=0.000179 \\
& \text { Formula 3: } \\
& \qquad p(z \mid y)=\frac{p(y, z)}{p(y)}=\frac{r(y, z)}{r(y)}=0.000426 \\
& \text { Formula 4: } \\
& \qquad \sigma^{2}\left(p(y \mid x)=\frac{r(x, y)}{r^{2}(x)}=2.95919 \mathrm{E}-11\right. \\
& \text { Formula 5: } \\
& \qquad \sigma^{2}\left(p(z \mid y)=\frac{r(y, z)}{r^{2}(y)}=4.54529 \mathrm{E}-08\right. \\
& \text { Formula 6: } \quad(p(z \mid y)-p(y \mid x)) \\
& t_{x, z(y)}=\frac{(p)}{\sqrt{\sigma^{2}\left(p(y \mid x)+\sigma^{2}(p(z \mid y)\right.}}=1.161095
\end{aligned}
$$

According to the value calculated above, we find that $\mathrm{t}_{\mathrm{x}, \mathrm{z}(\mathrm{y})}=1.161095>0$, which means "the complex houses" has a preferred structure in which [[complex]ADJ+[houses]N]NP is a prototype. The structure of $[[$ the $]$ DET $+[$ complex $]$ ADJ $] N P$ which should be the correct choice is unpreferred. The shift of processing from the preferred structure to the unpreferred structure brings the processing breakdown and backtracking. The pseudo processing of garden path sentence has been discussed above and now the genuine ultimate processing algorithm will be shown below.

\section{The Processing Algorithm of Garden Path Sentence}

The genuine processing of garden path sentence can be clearly shown in the matrix. Please see the Table 3 in which "V $\rightarrow$ \{houses\}" is parsed.
TABle 3 The Matrix OF Genuine Processing
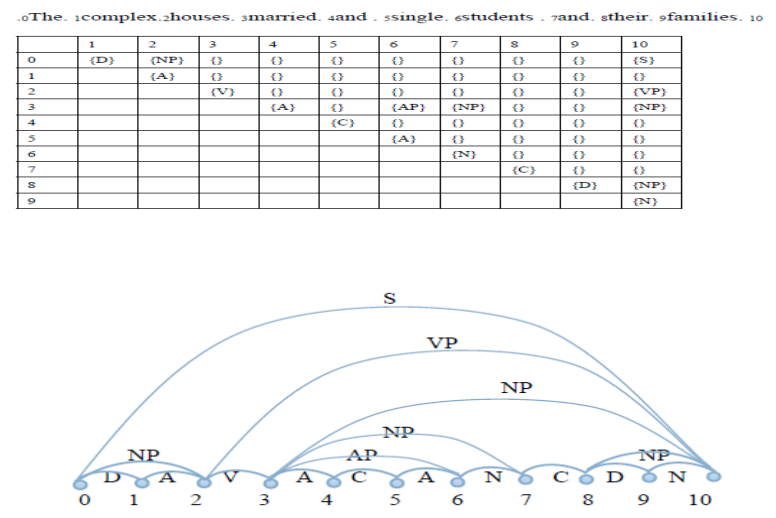

Figure 6 Sub-String Table of Genuine Processing for Example 3

In Table 3, we can find that all the words are parsed and the ultimate rule $\mathrm{S} \rightarrow \mathrm{NP}$ VP is well founded, which presents the successful parsing of the garden path sentence. Please see the well-formed sub-string table of genuine processing for Example 3.

The algorithm involved in the genuine processing of Example 3 can be clearly shown below.

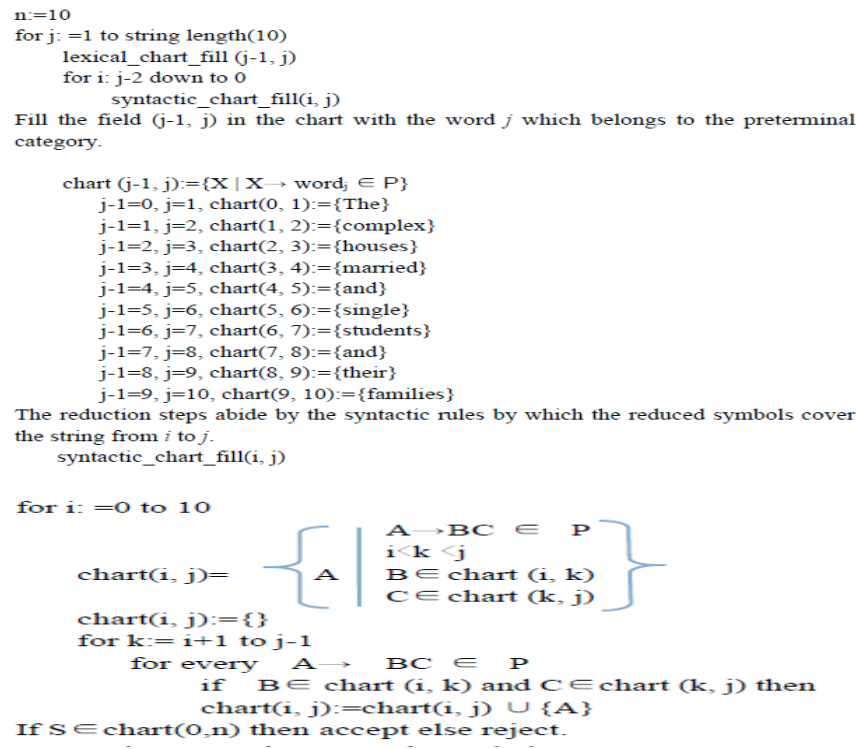

According to the algorithm, we can obtain the processing procedures in which all the elements are included in the chart.

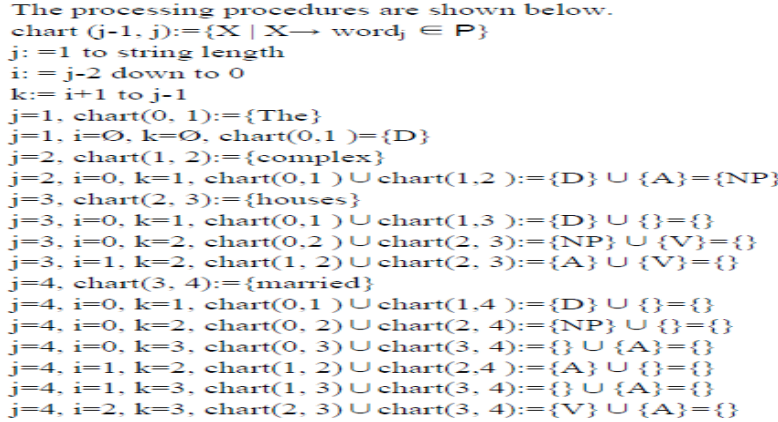

(Some steps of the procedure are omitted) 


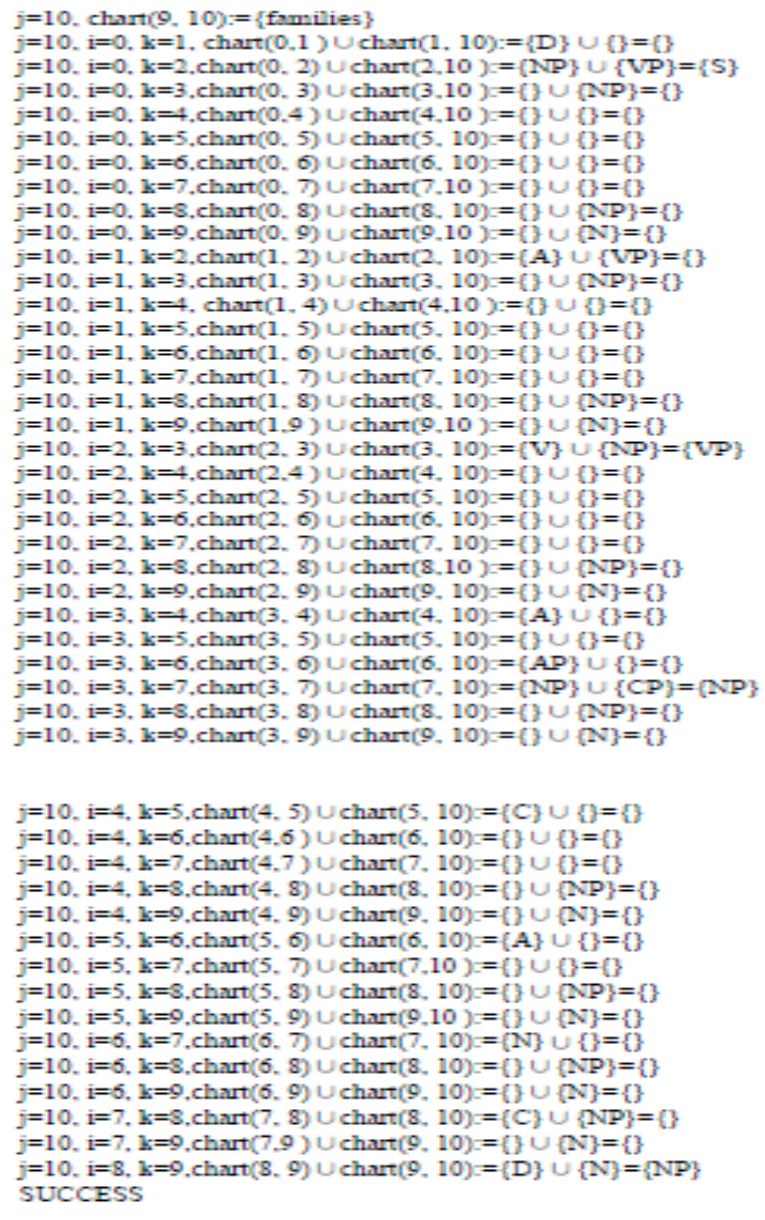

According to the processing procedure above, we can find that garden path sentence has a complex structure. The integration of the computational and linguistic knowledge is helpful for the processing of the complex sentence.

\section{CONCLUSION}

Machine learning (ML) system concerns the design of algorithms helpful for computational development and linguistic cognition. The automatic procedure focuses on the data mining and information filtering systems which are related to the automatic learning process on basis of the users' interests. The processing to the complex structure is an effective method to test ML system. The garden path sentences parsing include the initial pseudo processing and the ultimate genuine processing. The peculiarities of backtracking and breakdown involved in the pseudo processing require ML system efficient enough to work. The discussion in this paper reflects the fact that a hybrid technique of computational linguistics, including theoretical and statistical analysis, is an effective approach to parse garden path sentences for ML system.

\section{REFERENCES}

[1] X. Tang and M. Han, "Partial Lanczos extreme learning machine for single-output regression problems," Neurocomputing, vol.72, no. 13, pp. 3066-3076, 2009. http://dx.doi.org/10.1016/j.neucom. $\underline{2009.03 .016}$
[2] H. J. Rong, Y. S. Ong, A. H. Tan, et al, "A fast pruned-extreme learning machine for classification problem," Neurocomputing, vol. 72, no. 1, pp. 359-366, 2008. http://dx.doi.org/10.1016/j.neuc om.2008.01.005

[3] Bratko and T.Urbančič, "Transfer of control skill by machine learning," Eng. Appl. Artif. Int., vol.10, no 1, pp. 63-71, 1997. http://dx.doi.org/10.1016/S0952-1976(96)00076-0

[4] B. L. Whitehall, S. C. Y. Lu and R. E. Stepp, "CAQ: A machine learning tool for engineering," Artif. Int. Eng., vol. 5, no. 4, pp. 189-198, 1990. http://dx.doi.org/10.1016/0954-1810(90)90020-5

[5] T. Howley, M. G. Madden, M. L. O'Connell, et al, "The effect of principal component analysis on machine learning accuracy with high-dimensional spectral data," Knowl-Based Syst., vol. 19, no. 5, pp. 363-370, 2006. http://dx.doi.org/10.1016/j.knosys.2005.11.014

[6] G. B. Huang, X. Ding, H. Zhou, "Optimization method based extreme learning machine for classification," Neurocomputing, vol. 74, no. 1, pp. 155-163, 2010. http://dx.doi.org/10.1016/j.neucom. $\underline{2010.02 .019}$

[7] K. J. Cios and L. A. Kurgan, "CLIP4: Hybrid inductive machine learning algorithm that generates inequality rules," Inform. Sci., vol. 163 , no. 1 , pp. 37-83, 2004. http://dx.doi.org/10.1016/j.ins. 2003.03.015

[8] J. B. Wang, "Single-machine scheduling with general learning functions," Comput. \& Math. Appl., vol. 56, no. 8, pp.1941-1947, 2008. http://dx.doi.org/10.1016/j.camwa.2008.04.019

[9] Lykourentzou, I. Giannoukos, V. Nikolopoulos et al, "Dropout prediction in e-learning courses through the combination of machine learning techniques," Comput. \& Educat., vol. 53, no. 3, pp. 950-965, 2009.

[10] S. Stumpf, V. Rajaram, L Li et al, "Interacting meaningfully with machine learning systems: Three experiments," Int. J. HumComput. St., vol. 67, no. 8, pp. 639-662, 2009.

[11] E. Steiner, "Some remarks on a functional level for machine translation," Lang. Sci., vol. 14, pp. 607-621, October 1992. http://dx.doi.org/10.1016/0388-0001(92)90032-A

[12] J. Shin, P. G. Georgiou, and S. Narayanan, "Towards modeling user behavior in interactions mediated through an automated bidirectional speech translation system," Comput. Speech. Lang., vol. 24, pp. 232-256, April 2010. http://dx.doi.org/10.1016/ j.csl.2009.04.008

[13] Y. S. Hwang, A. Finch, and Y. Sasaki, "Improving statistical machine translation using shallow linguistic knowledge," Comput. Speech. Lang., vol. 21, pp. 350-372, April 2007. http://dx.doi.org/10.1016/j.csl.2006.06.007

[14] W. Ilg and K. Berns, "A learning architecture based on reinforcement learning for adaptive control of the walking machine LAURON," Robot. Auton. Syst., vol. 15, no. 4, pp. 321-334, 1995. http://dx.doi.org/10.1016/0921-8890(95)00009-5

[15] J. L. Du, "The asymmetric information compensation hypothesis: research on confusion quotient in garden path model", Doctoral Dissertation for Communication University of China, 2013.

\section{AUTHORS}

Dr. J. L. Du is with Lexicographical Research Center, Guangdong University of Foreign Studies, Guangzhou, China (e-mail: dujiali68@126.com).

Dr. P. F. Yu is with Faculty of Chinese Language and Culture, with Guangdong University of Foreign Studies, Guangzhou, China (e-mail: yupingfang68@126.com).

Dr. M. L. Li is with the School of Education and Professional Studies, Griffith University, Australia (email: minglin.li@hotmail.com).

This work was supported in part by China Post-Funded National Social Science Foundation under Grants 12FYY019 and 12FYY021. Submitted 11 August 2014. Published as resubmitted by the authors 08 December 2014. 